\title{
Validação do Inventánio de Concepções de Deficiência em Situações de Trabalho (ICD-ST)
}

\author{
Mania Nivalda deCarvalhoFrétas- UnivesidadeFedaal deSãoJoãodł-Rei, SãoJoãodd-Ré, Brasil
}

\begin{abstract}
Resumo
A análise e gestão das condições de inserção de pessoas com deficiência é uma nova necessidade nas organizações de trabalho em função da Lei de Cotas. 0 objetivo deste estudo foi construir e validar uma escala sobre as formas como as pessoas percebem a deficiência e as possibilidades de trabalho das pessoas com deficiência (PcDs). A escala intitulada "Inventário de Concepções de Deficiência em Situações de Trabalho" foi aplicada em 163 gerentes de 18 empresas brasileiras. Por meio da análise fatorial exploratória, geraram-se sete fatores: Espiritual, Normalidade, Inclusão, Desempenho, Benefícios, Vínculo e Treinamento. Os coeficientes de fidedignidade variaram de 0,53 a 0,81, com resultado de 0,69 para a escala total. Os fatores possuem sentido teórico, tendo como referências a literatura e a avaliação de juízes. A validação do ICD-ST fornece evidências de que diferentes formas de perceber a deficiência podem ser identificadas em gestores que atuam com pessoas com deficiência.
\end{abstract}

Palavras dhave Pessoas com deficiência; Concepções de deficiência; Validação de escala.

\section{Validation of the Inventory on Disability Conceptions in Work Situations (ICD-ST)}

\begin{abstract}
Analysis and management of insertion conditions of people with disabilities is a new challenge to workplaces due to the Quota Schemes. The purpose of this study was to design and validate a scale about the way how people realize the disability and the work possibilities of people with disability (PWD). The scale entitled Inventory of the Disability Conceptions in Work Situations was administered to 163 managers from 18 Brazilian enterprises. An exploratory factor analysis was carried out to get evidence on the construct validity. This analysis generated seven factors Spiritual, Normality, Inclusion, Performance, Benefits, Relationship and Training. The reliability coefficients varied from 0.53 to 0.81 ., with the result of 0.69 to the global scale. Factors have theoretical meaning considering the literature and expert judgment of the item's relevance. Validation brings evidence that different ways of realizing the disability can be identify among managers who work on PWD.
\end{abstract}

Kegnords People with disability; Disability conceptions; Scale validation.

\section{Validación del “Inventário de Concepções de Deficiência em Situações de Trabalho” (ICD-ST)}

\begin{abstract}
Resumen
El análisis y gestión de las condiciones de inserción de personas con deficiencia es una nueva necesidad en las organizaciones de trabajo en función de la Ley de Cuotas. El objetivo de este estudio fue construir y validar una escala sobre las formas como las personas perciben la deficiencia y las posibilidades de trabajo de las personas con deficiencia (PcDs). La escala intitulada "Inventário de Concepções de Deficiência em Situações de Trabalho" fue aplicada en 163 gerentes de 18 empresas brasileñas. Por medio del análisis factorial exploratorio, se generaron siete factores: Espiritual, Normalidad, Inclusión, D esempeño, Beneficios, Vínculo y Entrenamiento. Los coeficientes de fiabilidad variaron de 0,53 a 0,81, con resultado de 0,69 para la escala total. Los factores poseen sentido teórico, teniendo como referencias la literatura y la evaluación de jueces. La validación del ICD-ST provee evidencias de que diferentes formas de percibir la deficiencia pueden ser identificadas en gestores que actúan con personas con deficiencia.

Palabrasdave Personas con deficiencia; Concepciones de deficiencia; Validación de escala.
\end{abstract}

A inclusão pode ser associada à inserção física de pessoas excluídas ou sub-representadas nos locais de trabalho. No entanto, segundo Ferdman, Barrera, Allen e Voung (2009), a inclusão se refere tanto ao julgamento ou percepção de aceitação coletiva experienciada quanto aos comportamentos que promovam o sentimento de pertença. No caso das pessoas com deficiência (PcDs), implica a construção de políticas e práticas cotidianas que proporcionem a acessibilidade para que elas supram seus desejos e necessidades, efetivando a construção de sua cidadania, o que inclui a participação efetiva delas no mercado de trabalho. A acessibilidade envolve a eliminação das barreiras físicas, de comunicação e atitudinais. As primeiras dizem respeito às barreiras arquitetônicas e de mobiliários, que dificultam a mobilidade das PcDs. As barreiras de comunicação são aquelas que impedem uma plena comunicação interpessoal, escrita e virtual. Já as barreiras atitudinais referem-se aos valores e concepções sobre a PcD e sobre a deficiência e associam-se à discriminação, ao preconceito, aos estigmas e aos estereótipos (Vivarta, 2003).

Nesse cenário de discussão sobre as questões de inclusão e acessibilidade, as pesquisas têm demonstrado as dificuldades das pessoas com deficiência ${ }^{1}$ para se inserirem e se manterem no mercado de trabalho a

\footnotetext{
${ }^{1}$ Nesta pesquisa, a deficiênia é entendida como o produto ou a consequência da articulação entre a perda ou disfunção anatômica, fisiológica ou psicológica da PcD e as circunstâncias concretas da sociedade (desenvolvimento do conhecimento, espaços acessíveis, atitudes para com a diferença etc.). Como produto ou consequência, a deficiência se concretiza na maior ou menor autonomia da $\mathrm{PCD}$ e na intensificação ou não da discriminação social (Carvalho-Freitas, 2007).
} 
despeito da politica de cotas (Batista, 2004; Neri, Pinto, Soares \& Castilha, 2003; Ribeiro \& Carneiro, 2009), as dúvidas dos empregadores a respeito da inserção dessas pessoas no mercado de trabalho formal (CarvalhoFreitas, Toledo, Nepomuceno, Suzano \& Almeida, 2010; Oliveira, 1993) e a maior possibilidade de contratação dessas pessoas por parte de grandes empresas que possuam profissionais de Recursos Humanos (Goss, Goss \& Adam-Smith, 2000).

Diante da exigência de se contratarem PcD s para as organizações, em função da Lei de Cotas, foram buscadas, na literatura nacional e internacional, escalas que contemplassem questões referentes às barreiras atitudinais existentes no processo de inserção de PcDs no trabalho. Foi encontrado um modelo multidimensional, desenvolvido por Stone e Colella (1996), que analisa os fatores que afetam o tratamento de indivíduos com deficiência nas organizações. 0 modelo de análise trabalha com quatro dimensões: a legislação, as características organizacionais e os aspectos individuais (tanto das pessoas com deficiência como de colegas e supervisores), além da natureza do trabalho. Nos atributos das PcDs, são considerados, dentre outros, o gênero, a raça, o estilo interpessoal e 0 tipo de deficiência. A construção do modelo parte do pressuposto de que tanto os fatores pessoais quanto os ambientais influenciam no tratamento de pessoas com deficiência nas organizações. Além disso, características do observador (colegas e supervisores) e das PcDs influenciam os processos psicológicos do observador, incluindo categorização, construção de estereótipos e estados afetivos.

Foram encontradas, na literatura internacional, várias escalas que avaliam as atitudes em relação às PcDs, por exemplo: Escala de Fatores sobre a D eficiência (Siller, 1969), Escala de Reação Situacional e de Disposição em relação às PcDs (Berry \& Jones, 1991), Escala de Atitudes em Relação aos Indivíduos com D eficiência no Ambiente de Trabalho (Popovich, Scherbaum, Scherbaum \& Polinko, 2003) e escalas gerais de Atitudes em Relação às Pessoas com Deficiência (Antonak \& Livneh, 1988; Forlin, Fogarty \& Caroll, 1999). Essas escalas têm por foco crenças e reações afetivas relacionadas às características de comportamento atribuídas às PcDs. No Brasil, foi identificada uma escala denominada "Escala de Atitudes Sociais em Relação ao Trabalho de Pessoas com Deficiência" (Tanaka, 2007). No entanto, "os resultados obtidos entre duas aplicações apresentaram flutuações, não garantindo a sua fidedignidade" (p. 15).

Considerando que a deficiência tem sido contemplada de formas diferentes ao longo da história e que elas tiveram um impacto na inserção ou exclusão dessas pessoas tanto na sociedade quanto no trabalho
(C'arvalho-Freitas \& Marques, 2007), foi feita a opção por buscar construir uma escala que não naturalizasse a deficiência como algo circunscrito a uma dimensão física, e, sim, que a contemplasse a partir de uma perspectiva histórica.

Carvalho-Freitas e Marques (2007), por meio de uma análise histórica, identificaram maneiras específicas de conceber a deficiência (crenças) e se relacionar com as PcDs, que permaneciam as mesmas, embora 0 contexto histórico e social se modificasse. A partir dessa identificação, construíram uma tipologia que denominaram de matrizes de interpretação da deficiência. São elas:

1. A matriz da subsistência/ sobrevivência: é uma tipificação das formas de ver a deficiência, que tem na necessidade de sobrevivência e manutenção da sociedade seus principais fundamentos e possibilidade de entendimento dos padrões de comportamento em relação à $\mathrm{PcD}$. Associa a deficiência à dificuldade ou impossibilidade de participação social, aceitando apenas incluir uma PcD mediante a comprovação de sua contribuição efetiva para a comunidade.

2. A matriz da sociedade ideal e a função instrumental da pessoa: têm como fundamento de avaliação a busca de uma sociedade perfeita com foco na função das pessoas para a sociedade. A deficiência é vista como 0 antônimo do ideal de homem valorizado, e a exclusão das PcDs e seu extermínio, uma ação necessária para o equilíbrio social.

3. A matriz da deficiência como fenômeno espiritual: é uma modalidade de pensamento que tem na espiritualidade sua forma de avaliação predominante e associa a deficiência a uma origem espiritual, considerando-a como um desígnio transcendente, concretizado na relação divina para com o mundo, por meio da escolha das características que a pessoa deverá possuir durante sua vida. Como consequência desse pressuposto, as pessoas que compartilham dessa premissa reconhecem como forma legítima de lidar com as PcDs os comportamentos pautados na caridade e na compaixão, garantindo, desse modo, a inserção.

4. A normalidade como matriz de interpretação: as pessoas que compartilham desta concepção têm na normalidade sua referência de avaliação predominante das pessoas e associam a deficiência a problemas biológicos, anatômicos ou psicológicos, resultantes de disfunções genéticas ou de adoecimento. Consideram as pessoas que não se enquadram nos padrões de normalidade como desviantes. Pode ser denominada como modelo médico da deficiência (Goss \& colaboradores, 2000). A reabilitação das PcDs é considerada como uma questão das ciências da saúde e a adequação social como uma responsabilidade da própria pessoa. Em razão desses pressupostos, a 
inserção será decorrente do tipo e gravidade da deficiência e da possibilidade de adequação da PcD aos espaços sociais.

5. A inclusão como matriz de interpretação: esta concepção é compartilhada pelas pessoas que têm como referência de avaliação a garantia do direito de pertencimento social igualitário para todos e associam a deficiência a uma falha da sociedade em ser capaz de prover condições de exercício da cidadania para todas as pessoas, independente de suas diferenças. Pode também ser denominada como modelo social da deficiência (Barnes, 1999; Barnes, Oliver \& Barton, 2002). Em virtude dessa premissa, a inserção das PcDs será decorrente das possibilidades de adaptação da sociedade para ser acessível a todos.

6. A matriz de interpretação técnica da deficiência: as pessoas que compartilham desta concepção têm a funcionalidade das pessoas e sua capacidade produtiva como modalidade de pensamento predominante e avaliam as PcDs como um recurso a ser gerido nas organizações, considerando as práticas sociais e organizacionais como veículos para a participação e inclusão dessas pessoas. Esta matriz se traduz em percepções relacionadas ao trabalho propriamente dito da $\mathrm{PcD}$, principalmente no que se refere ao desempenho, vínculo dessas pessoas com a organização, benefícios para a organização e necessidade de treinamento.

Essas matrizes, por se constituírem em tipificações das diversas formas de percepção da deficiência, podem coexistir em uma mesma pessoa, pois, segundo Carvalho-Freitas e Marques (2007), elas são respostas possíveis à deficiência, que permanecem na atualidade. Por outro lado, existem duas perspectivas diferentes de interpretação da deficiência e das possibilidades das PcDs. De um lado, as matrizes espiritual, da normalidade e técnica, que têm por foco a deficiência; e, do outro, a matriz da inclusão, cujo foco é a modificação das condições da sociedade para ser acessível a todos. Considera-se que a maior concordância com as premissas da matriz da inclusão implicaria uma diminuição da concordância com as demais matrizes mencionadas.

Definiu-se, então, como objetivo geral deste estudo, desenvolver um Inventário de Concepções de Deficiência em Situações de Trabalho (ICD-ST), que pudesse auxiliar no diagnóstico e planejamento de ações voltadas para a análise das crenças das pessoas sobre a deficiência e as possibilidades de trabalho das PcD s. Assim, procurou-se: a) operacionalizar as formas de ver a deficiência ao longo da história, identificadas por Carvalho-Freitas e Marques (2007); b) verificar as características psicométricas do instrumento construído; c) analisar se a estrutura fatorial encontrada na escala envolve dimensões de análise significativas; d) identificar, quanto às diversas maneiras de perceber a deficiência, possíveis diferenças entre sexos, faixas etárias e tipos de empresa; e) avaliar se existe correlação entre tempo de trabalho com PcDs e os fatores de concepção de deficiência. No estudo realizado, foram contempladas pessoas com deficiência física, auditiva e visual, sem comprometimento intelectual.

\section{Método}

Tanto na construção da escala quanto em sua validação, houve a participação de segmentos da população à qual se pretende destinar o instrumento. Para atingir os objetivos da pesquisa, são descritos, a seguir, os procedimentos adotados para a construção e para a realização da validade de conteúdo do instrumento, além da avaliação da precisão do mesmo.

\section{Participantes}

Devido às dificuldades de informação em relação às empresas que contratam ou não pessoas com deficiência, o universo considerado para a realização da pesquisa foi 147 empresas que, no ano de 2004, publicaram seu balanço social no Instituto Brasileiro de Análises Sociais e Econômicas (IBASE). Essas empresas se constituíam na totalidade das empresas que contratavam $\mathrm{PcD}$ s e que tinham seus resultados disponibilizados, por via eletrônica, nesse Instituto. D essas empresas, 18 efetivamente contribuíram com a pesquisa.

0 método de pesquisa utilizado foi o survey de desenho transversal. Os questionários foram administrados por meio do método eletrônico de survey pela inteme. O ICD-ST foi aplicado a uma amostra de conveniência composta por 18 empresas e 163 gestores. A composição final da amostra está explicitada na Tabela 1.

A Tabela 1 mostra que $78 \%$ das empresas participantes são do setor de serviços (bancos, empresas de comunicação, transporte e outros). Em Outros, constam: uma empresa de serviço ao consumidor, uma usina hidrelétrica, uma empresa de distribuição e uma empresa de pesquisas. D as empresas participantes, 83\% possuem mais de mil funcionários em seu quadro de pessoal. Com relação ao número de pessoas com deficiência, 39\% delas possuem entre 20 e 100 funcionários com deficiência e 28\% possuem entre 100 e 500 funcionários com deficiência. Também fizeram parte da amostra $28 \%$ de empresas que possuem até 20 funcionários com deficiência e $5 \%$ que possuem mais de 1.000 funcionários com deficiência. 
Tabela 1. Caracterização das empresas participantes da pesquisa

\begin{tabular}{|c|c|c|c|c|c|c|c|c|c|c|}
\hline \multirow[b]{2}{*}{$\begin{array}{c}\text { Setor de } \\
\text { Atividade } \\
\text { (segmento) }\end{array}$} & \multirow[b]{2}{*}{$\begin{array}{l}\text { Número } \\
\text { de } \\
\text { empresas }\end{array}$} & \multicolumn{3}{|c|}{ Número de funcionários } & \multicolumn{5}{|c|}{ Número de pessoas com deficiência } & \multirow[b]{2}{*}{$\begin{array}{l}\text { Número de } \\
\text { participantes } \\
\text { da pesquisa }\end{array}$} \\
\hline & & $\begin{array}{c}201 \\
\mathrm{a} \\
500\end{array}$ & $\begin{array}{c}501 \\
\mathrm{a} \\
1.000\end{array}$ & $\begin{array}{c}\text { Acima } \\
\text { de } \\
1.000\end{array}$ & $\begin{array}{c}\text { Até } \\
20\end{array}$ & $\begin{array}{c}21 \\
a \\
50\end{array}$ & $\begin{array}{c}51 \\
\mathrm{a} \\
100\end{array}$ & $\begin{array}{c}101 \\
\mathrm{a} \\
500\end{array}$ & $\begin{array}{c}\text { Acima } \\
\text { de } \\
1.000\end{array}$ & \\
\hline Agrícola & 01 & & & 01 & & & 01 & & & 02 \\
\hline Indústria & 03 & & 01 & 02 & 02 & & & 01 & & 08 \\
\hline Bancos & 03 & & & 03 & 02 & & & & 01 & 121 \\
\hline Comunicação & 03 & 01 & & 02 & & 01 & & 02 & & 09 \\
\hline Transporte & 04 & & 01 & 03 & 01 & 01 & 01 & 01 & & 15 \\
\hline Outros & 04 & & & 04 & & 02 & 01 & 01 & & 08 \\
\hline Total & 18 & 01 & 02 & 15 & 05 & 04 & 03 & 05 & 01 & 163 \\
\hline
\end{tabular}

Fonte: D ados da Pesquisa.

Três empresas participantes têm unidades em todo o território nacional e as demais se distribuem em 16 estados do país, sendo que três delas estão distribuídas em dois ou mais estados. Os participantes (unidades de observação) da pesquisa foram: profissionais que se responsabilizavam pela inserção de PcDs nas empresas (a maioria da área de Recursos Humanos), profissionais da área de Saúde e Segurança no Trabalho e chefias imediatas das pessoas com deficiência, num total de 163 pessoas. A média de idade dos participantes foi de 43 anos. Eram do sexo masculino $73 \%$ deles e tinham formação de nível superior 91\% do total de participantes. Esses gerentes trabalham há 3,6 anos em média com PcDs, embora $40 \%$ da amostra trabalhem há menos de um ano com elas (Moda = 1 ano).

\section{Instrumento}

Para a construção do Inventário de Concepções de Deficiência, foi deslocada a observação dos atributos das PcDs e das reações provocadas pela deficiência para a categorização de formas de ver a deficiência ao longo da história. $\mathrm{O}$ objetivo foi desnaturalizar a questão da deficiência, como um atributo característico das PcDs, e recolocar as formas de ver e se relacionar com as pessoas com deficiência na dimensão das relações historicamente produzidas.

Foram utilizadas quatro matrizes de interpretação da deficiência para a construção do ICD -ST a partir da revisão histórica realizada por Carvalho-Freitas e Marques (2007): a matriz espiritual, a matriz da normalidade, a matriz da inclusão e a matriz técnica. As duas primeiras matrizes não foram operacionalizadas, nesta pesquisa, para o desenvolvimento do ICD-ST: a matriz da sobrevivência/ subsistência, por trazer, implícitos, aspectos que também são compartilhados pelas demais matrizes e que dizem respeito à busca de manutenção da sociedade, principalmente quando relacionados às situações de trabalho; a segunda, denominada "sociedade ideal e a função instrumental da pessoa", por refletir pressupostos rechaçados pela sociedade moderna, sendo que pouco provavelmente as pessoas assumiram possuí-los, principalmente nas organizações de trabalho.

O Inventário de Concepções de Deficiência em Situações de Trabalho foi composto inicialmente por 27 itens, tendo sido submetido a um painel de especialistas (juízes) composto por: três pesquisadores do tema inserção social de pessoas com deficiência, um professor de metodologia científica, um pesquisador sobre saúde e segurança no trabalho, dois doutorandos em administração, dois profissionais de recursos humanos que atuavam com inserção de pessoas com deficiência e seis alunos de graduação em administração que trabalhavam em empresas (públicoalvo do inventário). Esses juízes avaliaram a pertinência dos itens em cada dimensão e sugeriram modificações no inventário (validade de construto), conforme orientações de Pasquali (2009). Foi solicitado a cada especialista que avaliasse, individualmente, a qual dos fatores cada item do inventário se referia, tendo sido formecida a cada um deles duas listagens: uma, contendo a relação dos fatores e sua definição; e outra, de dupla entrada, em que, nas linhas, foram elencados todos os itens do inventário e, nas colunas, os possíveis fatores aos quais pertenciam. Os juízes marcaram 0 fator ao qual julgavam que cada item pertencia. Os itens mantidos foram aqueles que tiveram uma concordância maior que $80 \%$ entre os juízes.

O inventário foi, então, aplicado em uma amostra pré-teste (análise semântica) composta por 137 alunos de especialização em administração e submetido à análise fatorial com vistas a purificar a escala. Foram, assim, a partir das análises realizadas, acrescentados itens no questionário original e retirados aqueles que não apresentaram carga acima de 0,30 nos fatores encontrados. 0 questionário passou de 27 para 34 itens. 0 questionário definitivo foi composto por questões fechadas, com a utilização da escala Liket, de escolha forçada, para medição, considerando-se uma variação de 1 (discorda totalmente) a 6 (concorda totalmente). 


\section{Procedimentos}

A pesquisa foi aprovada pelo Comitê de Ética da universidade e os procedimentos adotados para a validação dos instrumentos de pesquisa foram realizados conforme orientações de Pasquali (1999). A dimensionalidade do Inventário de Concepções de D eficiência foi estabelecida por meio da análise fatorial.

Em relação à amostra obtida nas empresas que trabalhavam com pessoas com deficiência, 0 tratamento preliminar dos dados identificou a ocorrência de menos de 5\% de casos faltosos (missing valuess), os quais foram eliminados das análises. Todos os itens do inventário foram mantidos nas análises, pois apresentaram carga fatorial superior a 0,30 e nenhuma ambiguidade. 0 índice de Kaiser-MeyerOlkin $(\mathrm{KMO}=0,660)$ e 0 teste de esfericidade de Bartlett (significativo a $\mathrm{p}<0,001$ ) indicaram a fatorabilidade dos dados. A amostra conseguida para a realização da análise fatorial do ICD-ST foi de cinco respondentes para cada variável. Segundo Hair, Anderson, Tatham e Black (2005), "como regra geral, o mínimo é ter pelo menos cinco vezes mais observações do que 0 número de variáveis a serem analisadas" ( $p$. 98).

Foi realizada a análise de componentes principais, pois o objetivo era determinar o número mínimo de fatores que respondessem pela máxima variância dos dados. Depois, foi realizada a análise de fatores comuns, porque se buscava identificar as dimensões ou construtos latentes, sendo a solução de fatores semelhante à fornecida pela análise de componentes principais. Foram feitas a rotação ortogonal varimax e a rotação oblíqua oblimin. Como os elementos fora da diagonal principal da matriz de correlação dos fatores (fator comdation matrix) da rotação oblíqua foram todos menores do que 0,30 e a matriz de padrões (pattem matrix) apresentou cargas fatoriais muito próximas das obtidas com a rotação ortogonal, optou-se pela estrutura fatorial mais simples, a ortogonal, conforme sugerida por Tabachnick e Fidell (1996).

\section{Resultados}

Os resultados estatísticos da pesquisa indicaram, em princípio, a existência de 11 fatores com égenvalue superior a 1,0 , os quais explicavam $66 \%$ da variância total. Ao realizar a análise da precisão do instrumento, por meio do método de consistência interna (coeficiente alfa de Cronbach), dos 11 fatores encontrados, seis apresentaram consistência interna superior a 0,60, o que, de acordo com Hair e colaboradores (2005), indica confiabilidade satisfatória da consistência interna em pesquisa exploratória. Contudo, um fator com alfa de Cronbach igual a 0,53 foi mantido nas análises devido à sua relevância teórica para a construção do instrumento (Fator 4). O inventário foi reduzido a sete fatores com 21 itens, conforme Tabela 2.

A Tabela 2 mostra os pressupostos utilizados nas diferentes matrizes de interpretação da deficiência e sua operacionalização em indicadores (afirmativas do questionário), além de seu agrupamento nos fatores. É importante sublinhar que a matriz técnica se subdividiu, após a análise fatorial, em quatro fatores: desempenho, vínculo, benefícios da contratação e avaliação da necessidade de treinamento para a inserção de PcDs.

Tabela 2. D os construtos teóricos aos itens de operacionalização do ICD -ST

\begin{tabular}{|c|c|c|}
\hline Matriz & Definição de deficiência & Operacionalização por meio dos itens do questionário \\
\hline $\begin{array}{l}\text { Espiritual } \\
\text { Fator } 1\end{array}$ & $\begin{array}{l}\text { Deficiência vista como fenômeno } \\
\text { espiritual, atribuindo origem } \\
\text { metafísica à deficiência e a } \\
\text { considerando uma manifestação } \\
\text { de desejos ou castigos divinos. } \\
\text { Conduta predominante: caridade. }\end{array}$ & $\begin{array}{l}\text { A deficiência é uma manifestação de um poder divino que define as } \\
\text { caracteństicas que o ser humano deverá possuir em sua vida terrena. } \\
\text { A deficiência é uma marca que indica uma preferência divina. } \\
\text { As pessoas com deficiência têm uma missão especial em suas vidas. }\end{array}$ \\
\hline $\begin{array}{l}\text { Normali- } \\
\text { dade } \\
\text { Fator } 2\end{array}$ & $\begin{array}{l}\text { Tem na norma seu padrão de } \\
\text { avaliação, sendo a deficiência } \\
\text { considerada um "desvio" ou } \\
\text { "doença" que necessita de } \\
\text { cuidados especiais. Considera } \\
\text { adequada a segregação das PcD s } \\
\text { em espaços especificos. Modelo } \\
\text { "médico" da deficiência. }\end{array}$ & $\begin{array}{l}\text { Somente alguns setores são adequados para o trabalho das PcDs. } \\
\text { As pessoas com deficiência podem assumir atitudes inadequadas na } \\
\text { empresa, provocando situações embaraçosas. } \\
\text { As PcD s são mais propensas a se acidentar que as demais pessoas. } \\
\text { As instituições especializadas são mais aptas a trabalhar com pessoas com } \\
\text { deficiência que as empresas. } \\
\text { As pessoas com deficiência costumam ter problemas no relacionamento } \\
\text { com as pessoas sem deficiência. }\end{array}$ \\
\hline
\end{tabular}

Fonte: Elaborada a partir da revisão de Carvalho-Freitas e Marques (2007). 
Tabela 2. D os construtos teóricos aos itens de operacionalização do ICD-ST

\begin{tabular}{|c|c|}
\hline Matriz & Definição de deficiência \\
\hline Inclusão & $\begin{array}{l}\text { Desloca a deficiência de } \\
\text { problema individual para } \\
\text { um }\end{array}$ \\
\hline Fator 3 & $\begin{array}{l}\text { problema social. Seu pressuposto } \\
\text { é de que a sociedade tem que se } \\
\text { adequar a todos e incluir } \\
\text { diferenças. Modelo social } \\
\text { deficiência }\end{array}$ \\
\hline Técnica & $\begin{array}{l}\text { A interpretação técnica da } \\
\text { deficiência se materializa quando } \\
\text { a diversidade passa a ser um }\end{array}$ \\
\hline & $\begin{array}{llr}\text { recurso a ser gerido pelas } \\
\text { organizações. } & \text { Reflete nas } \\
\text { avaliações sobre } 0 \text { trabalho das } \\
\text { PcDs nas orcanizacões. }\end{array}$ \\
\hline
\end{tabular}

Operacionalização por meio dos itens do questionário tipo de trabalho, desde que modificadas as condições de trabalho.

As pessoas com deficiência podem desempenhar adequadamente qualquer tipo de trabalho, desde que realizadas as adequações necessárias nos instrumentos de trabalho. diferenças. Modelo social da deficiência

Fator 4 - Desempenho

A qualidade do trabalho de uma pessoa com deficiência é inferior ao das pessoas sem deficiência.

As pessoas com deficiência desempenham o trabalho com baixa produtividade.

As pessoas com deficiência têm pior desempenho no trabalho.

As empresas com grande número de pessoas com deficiência são menos competitivas que as demais.

Fator 5 - Vínculo

As pessoas com deficiência são mais comprometidas que as demais.

As pessoas com deficiência são mais estáveis no emprego que as demais.

Fator6 - Benefícios

A convivência com pessoas com deficiência melhora o clima da empresa.

Contratar pessoas com deficiência melhora a imagem da empresa junto aos clientes.

Contratar pessoas com deficiência melhora a imagem da empresa junto aos funcionários.

Fator 7 - Treinamento

As chefias não estão preparadas para supervisionar o trabalho de pessoas com deficiência.

Os funcionários precisam ser treinados para trabalhar com pessoas com deficiência.

Fonte: Elaborada a partir da revisão de Carvalho-Freitas e Marques (2007).

Tabela 3. Matriz fatorial dos itens do Inventário de Concepções de D eficiência em situações de trabalho

\begin{tabular}{|c|c|c|c|c|c|c|c|}
\hline$\overline{\text { Item }}$ & Fator 1 & Fator 2 & Fator 3 & Fator 4 & Fator 5 & Fator 6 & Fator 7 \\
\hline 01 & 0,865 & & & & & & \\
\hline 02 & & & & & & & \\
\hline 03 & 0,478 & & & & & & \\
\hline 04 & & 0,521 & & & & & \\
\hline 05 & & 0,680 & & & & & \\
\hline 06 & & 0,598 & & & & & \\
\hline 07 & & 0,389 & & & & & \\
\hline 08 & & 0,438 & & & & & \\
\hline 09 & & & 0,486 & & & & \\
\hline 10 & & & 0,821 & & & & \\
\hline 11 & & & & 0,677 & & & \\
\hline 12 & & & & 0,722 & & & \\
\hline 13 & & & & 0,764 & & & \\
\hline 14 & & & & 0,609 & & & \\
\hline 15 & & & & & 0,867 & & \\
\hline 16 & & & & & 0,737 & & \\
\hline 17 & & & & & & 0,674 & \\
\hline 18 & & & & & & 0,822 & \\
\hline 19 & & & & & & 0,839 & \\
\hline $\begin{array}{l}20 \\
21\end{array}$ & & & & & & & 0,815 \\
\hline Eigenvalues & 1,739 & 1,165 & 1,665 & 5,312 & 2,395 & 3,244 & 1,281 \\
\hline \% Variância Explicada & 5,116 & 3,426 & 4,896 & 15,623 & 7,044 & 9,540 & 3,767 \\
\hline
\end{tabular}

Fonte: D ados da pesquisa. 
A consistência interna também foi calculada para todo o inventário, apresentando um alfa de Cronbach no valor de 0,69. Esses sete fatores explicam $49 \%$ da variância total, o que representa um bom resultado em um estudo exploratório (Pasquali, 1999). Os fatores foram denominados segundo sua característica geral e sua relação com as matrizes de interpretação identificadas na introdução. Na Tabela 3, é apresentada a matriz fatorial dos Itens do Inventário de Concepções de Deficiência, composta pelos itens que permaneceram agrupados pelas dimensões às quais pertencem, bem como seus eignnalues e os valores da variância explicada.

A partir do agrupamento dos fatores apresentados na Tabela 3, pode-se inferir, como característica geral dos fatores, os seguintes aspectos: 0 fator expinitual focaliza a percepção da deficiência como um fenômeno espiritual. A predominância de alto grau de concordância neste fator pode indicar uma atitude de caridade em relação às PcDs e conflitos para os gestores no que se refere à avaliação do desempenho dessas pessoas, conforme identificado por CarvalhoFreitas (2009), o que também poderia dificultar as relações de trabalho devido às possíveis diferenciações no tratamento destinado aos trabalhadores com e sem deficiência.

O fator nomalidade focaliza a percepção das pessoas, tendo por referência o "desvio" da normalidade das PcDs e suas implicações para 0 trabalho, tais como atitudes inadequadas provocando situações embaraçosas, propensão a acidentes, alocação em setores específicos e problemas de relacionamento. Além disso, avalia a receptividade para a inserção de PcDs ao considerar as instituições especializadas mais adequadas ao trabalho dessas pessoas. A predominância de alto grau de concordância com este fator pode indicar uma tendência a segregar PcDs em setores específicos da organização, além de uma avaliação negativa das possibilidades de trabalho dessas pessoas.

0 fator indusão focaliza a percepção quanto à necessidade de adequação dos instrumentos e condições de trabalho para a inserção de PcDs na empresa. Um resultado alto neste fator indica uma percepção positiva em relação às possibilidades de trabalho das pessoas com deficiência e a consciência da necessidade de adequação das condições e instrumentos de trabalho, visando a dar condições de igualdade para todos.

O fatar desempenhofocaliza a percepção das pessoas em relação ao desempenho, produtividade e qualidade de trabalho das PcDs e suas implicações para a competitividade da empresa. Um alto resultado neste fator indica uma percepção negativa em relação ao desempenho e à qualidade de trabalho de uma pessoa com deficiência.

O fator benfíios focaliza a percepção do impacto da contratação de PcDs para a imagem da empresa junto a funcionários e clientes e também para 0 clima da organização. A predominância de alto grau de concordância neste fator indica uma percepção positiva do impacto da inserção de pessoas com deficiência para a imagem e 0 clima da organização.

O fator únaulofocaliza a percepção das pessoas em relação ao comprometimento e estabilidade no emprego das PcDs. A predominância de alto resultado neste fator indica uma percepção mais positiva do comprometimento e da estabilidade no emprego por parte de PcDs do que das demais pessoas

0 fator treinamento focaliza a percepção quanto à necessidade de treinamento das chefias e funcionários para a inserção de PcD s na empresa. Um alto resultado neste fator indica a percepção de necessidade de treinamento tanto das chefias quanto dos funcionários para a inserção de pessoas com deficiência na organização.

0 número de itens, a média, o desvio padrão de cada dimensão, bem como seus índices de fidedignidade (alfa de Cronbach) são apresentados na Tabela 4. Cabe ressaltar que a média foi calculada pelo somatório total dividido pelo número de itens, uma vez que cada fator apresenta diferenças na quantidade dos itens que o compõe.

Verifica-se, na Tabela 4, que os maiores índices de concordância da amostra pesquisada são os relacionados ao fator inclusão e aos fatores de desempenho e de benefícios da contratação (matriz técnica), avaliados como positivos pelos gestores. Os índices de fidedignidade encontrados podem ser considerados satisfatórios para um estudo exploratório. Conforme Hair e colaboradores (2005), um valor de alfa de Cronbach de 0,60 é aceitável neste tipo de pesquisa. Um dos possíveis motivos desses valores é 0 pequeno número de itens de cada fator.

Para investigar os efeitos do sexo, da faixa etária e do tipo de atividade da empresa (segmento) nos resultados médios dos fatores avaliados, foi utilizada a análise de variância (ANOVA), técnica estatística utilizada para verificar diferenças entre médias de duas ou mais populações. As médias dos fatores serão consideradas iguais quando o valor-p do teste for superior a 0,05. Não foram identificadas diferenças significativas entre as médias dos fatores de concepções de deficiência em nenhum dos aspectos analisados. 
Tabela 4. Propriedades das dimensões (fatores) do ICD-ST

\begin{tabular}{lcccc}
\hline Propriedade & Item $(\mathrm{n})$ & Média & Desvio padrão & Alfa de Cronbach \\
\hline 1. Espiritual & 3 & 2,21 & 1,09 & 0,64 \\
2. Normalidade & 5 & 2,71 & 0,84 & 0,65 \\
3. Inclusão & 2 & 4,71 & 0,96 & 0,53 \\
4. Desempenho & 4 & 5,28 & 0,75 & 0,77 \\
5. Vínculo & 2 & 3,34 & 1,34 & 0,79 \\
6. Benefícios & 3 & 4,86 & 0,87 & 0,81 \\
7. Treinamento & 2 & 3,84 & 1,19 & 0,62 \\
Escala Total & 21 & - & - & 0,69 \\
\hline
\end{tabular}

Fonte: Dados da Pesquisa.

Obs.: O s resultados dos itens de desempenho foram invertidos para facilitar as análises, mantendo a mesma coerência entre os fatores. Isto é, quanto maiores os resultados, maior a concordância positiva com o fator.

Buscando verificar um possível efeito do tempo de trabalho com PcDs e os fatores de concepção de deficiência, constatou-se que 0 fator percepção do desempenhoapresentou correlação positiva com o tempo de trabalho com pessoas com deficiência (correlação Speaman igual a 0,205, significativa no nível de 0,05). Esse resultado indica que há uma tendência em se ter uma percepção mais positiva do desempenho das PcD s com o passar do tempo de trabalho com elas.

\section{Discussão}

O objetivo deste estudo foi desenvolver um inventário que pudesse contemplar as formas de ver a deficiência, intitulado Inventário de Concepções de D eficiência em Situações de Trabalho (ICD-ST), assim como apresentar informações sobre as propriedades psicométricas do instrumento.

Verificou-se, então, que a estrutura fatorial do inventário envolve dimensões de análise significativas sobre a deficiência e sobre as possibilidades de trabalho das PcDs na percepção das pessoas que atuam com elas (PcDs). Os fatores extraídos: fator expinitual, fator nomalidade, fator indusãa fator desemperho fator únaulo, fator benfícios e fator treinamentopossuem sentido teórico, tendo como referências a literatura apresentada e a avaliação de juízes. Para cada um desses fatores foram encontradas consistências internas satisfatórias, considerando a natureza exploratória da pesquisa.

Os resultados referentes às variáveis sociodemográficas: sexo, faixa etária e setor de atividade da empresa não apresentaram diferenças. Um dado sociodemográfico que necessitaria ser avaliado e que, em função do perfil da amostra, não pôde ser analisado é a escolaridade dos gerentes e sua possível relação com as concepções de deficiência. Também seria interessante verificar se empresas que não divulgam seus resultados em balanços sociais teriam resultados similares aos da amostra pesquisada.

No entanto, foi verificada a presença de correlação entre o tempo de trabalho com PcDs e a percepção do desempenho dessas pessoas, indicando que quanto maior o tempo de trabalho com PcDs, melhor a percepção em relação às suas possibilidades de trabalho (desempenho). Esse resultado é coerente com a literatura, que tem indicado que o trabalho com PcDs favorece uma percepção positiva de suas possibilidades de desempenho (Gil, 2002) por permitir, principalmente, a personalização do relacionamento, avaliando as pessoas por suas qualidades particulares, $\mathrm{e}$ não por sua pertença a um grupo específico, como 0 caso das PcD s (Miller, 2002).

\section{Considerações finais}

O instrumento apresentado fornece evidências de que diferentes formas de ver a deficiência podem ser identificadas em gestores que atuam com PcDs, principalmente se se consideram as evidências de validade e fidedignidade apresentadas. 0 inventário indica a percepção das pessoas em relação aos diversos fatores analisados, permitindo verificar o que pensa cada uma delas sobre a deficiência e as possibilidades de trabalho das PcDs. Nesse sentido, o inventário mostra a tendência predominante de avaliação das pessoas. Diversas formas de ver a deficiência podem coexistir em uma mesma pessoa, denunciando a complexidade da temática e a necessidade de conhecimento e reflexão sobre a questão da deficiência. No entanto, o inventário permite identificar se existe uma tendência voltada mais para a inclusão, para a avaliação das PcD s a partir de critérios da normalidade ou tendo por foco a funcionalidade delas no trabalho. Além disso, o inventário permite discriminar diferenças entre as pessoas a partir de critérios sociodemográficos, como no caso do tempo de trabalho com as PcDs e a questão da avaliação do desempenho delas, como verificado na presente pesquisa.

Novos estudos serão necessários para verificar, por exemplo, resultados de estabilidade temporal do instrumento. Além disso, é necessário relacionar as formas de os gestores verem a deficiência, identificadas 
pelo ICD -ST, com aspectos referentes à percepção de adequação das condições e práticas de trabalho, com políticas e práticas de recursos humanos adotadas pelas empresas e com fatores relacionados aos valores organizacionais, dentre outros, tentando identificar relações teóricas que auxiliem na compreensão do processo de inserção de PcDs nas organizações de trabalho. Por fim, o Inventário de Concepções de Deficiência também poderá ser utilizado como instrumento para buscar modificações nas atitudes dos gestores a partir da reflexão sobre suas concepções de deficiência.

\section{Referências}

Antonak, R. F., \& Livneh, H. (1988). Measuremett of attitudes toward peeple with disabilities mothoos, psychomeries and scales Springfield: C. C. Thomas.

Barnes, C. (1999). D isability studies: new or not so new directions? Disability\& Socity, 14(4), 577-580.

Barnes, C., Oliver, M., \& Barton, L. (2002). Disability studiestoday. Cambridge: Polity Press.

Batista, C. A. M. (2004). Indusãa construçãona divesidade Belo Horizonte: Armazém de Idéias.

Berry, J. O., \& Jones, W. H. (1991). Situational and dispositional components of reactions toward persons with disabilities. The Jamal of Social Psychdogy, 131(5), 673-684.

Carvalho-Freitas, M. N. (2007). A inseção depesscas com deficiêna em empresas brasileras (Tese de D outorado). Universidade Federal de Minas Gerais, Belo Horizonte, Minas Gerais.

Carvalho-Freitas, M. N. (2009). Inserção e gestão do trabalho de pessoas com deficiência: um estudo de caso. RAC - Revista deAdministração Contemparânet, 13(Edição Especial), 121-138.

Carvalho-Freitas, M. N., \& Marques, A. L. (2007, abril/junho). A diversidade através da história: a inserção no trabalho de pessoas com deficiência. Organizaçós eSciedade(UFBA), 14(41), 59-78.

Carvalho-Freitas, M. N., Toledo, I. A., Nepomuceno, M. F., Suzano, J. C. C., \& Almeida, L. D . (2010, julho/ setembro). Socialização organizacional de pessoas com deficiência. RAE - Reista de AdministraçãodeEmpresas, 50(3), 264-275.

Ferdman, B. M., Barrera, V., Allen, A. A., \& Vuong, V. (2009). Indusivebehavior and the experience of indusion. Gender and dvesity in organizations dvision Trabalho apresentado na Annual Meeting of the Academy of Management, Philadelphia.
Forlin, C., Fogarty, G., \& Caroll, A. M. (1999). Validation of the factor structure of the Interactions with Disabled Persons Scale. A ustralian Jamal of Psydhogy, 51(1), 50-55.

Gil, M. (Coord.). (2002). O que as empres podem fazer pala indusão das pesscas com deficiênia. São Paulo: Instituto Ethos.

Goss, D., Goss, F., \& Adam-Smith, D. (2000). Disability and employment: a comparative critique of UK legislation. TheIntemational Jaumal of Human ResarceManagment, 11(4), 807-821.

Hair Jr., J. F., Anderson, R. E., Tatham, R. L., \& Black, W. C. (2005). Análise miltivaniada de dados (5 $5^{\underline{a}}$ ed.). Porto Alegre: Bookman.

Miller, N. (2002). Personalization and the promise of contact theory. Jamal of Social Issues, 58(2), 387410.

Neri, M., Pinto, A., Soares, W., \& Castilha, H. (2003). Reratos da deiciênia mo Brasil Rio de Janeiro: FGV/ IBRE, CPS.

Oliveira, M. H. A. (1993). Núcleos cooperativos: uma perspectiva profissional para 0 portador de deficiência. EmAbato 13(60), 106-108.

Pasquali, L. (O rg.). (1999). Instrumentos psiclógicos manual práticodedabaraçãa Brasília: LabPAM.

Pasquali, L. (2009). Psicommia: teeria dos testes na psicdoga ena đuração(3aed.). Petrópolis: Vozes.

Popovich, P. M., Scherbaum, C. A., Scherbaum, K. I., \& Polinko, N. (2003). The assessment of attitudes toward individual with disabilities in the workplace. TheJaumal of Psydhdogy, 137(2), 163-177.

Ribeiro, M. A., \& Carneiro, R. (2009, julho/ setembro). A inclusão indesejada: as empresas brasileiras face à lei de cotas para pessoas com deficiência. O\&SRevista Organização e Sociedade(UFBA), 16(50), 545564.

Siller, J. (1969). The general form of the Disability Factor Scales Series (DFS-G). Nova Iorque: New York University School of Education.

Stone, D. L., \& Colella, A. (1996, April). A model of factors affecting the treatment of disabled individuals in organizations. Acadamy of Managemet Review 21, Issue 2.

Tabachnick, B. G., \& Fidell L. S. (1996). Using miltivariate statistics ( $3^{\underline{a}}$ ed.). Boston: Pearson Education. 
Tanaka, E. D. O . (2007). O desendvimento deuma scala de atitudes soiais em redação ao trabalho da pessca cm deficênia (Tese de Doutorado). Universidade Estadual Paulista Júlio de Mesquita Filho, Marília, São Paulo.
Vivarta, V. (Coord.). (2003). Mídia e deficiêna (Série Diversidade). Brasília: Andi, Fundação Banco do Brasil.

Reedidoem16/ 02/ 2011

Refamiladoem02/ 11/ 2011

Aprovado em06/ 03/ 2012

Sobre a autora:

Maria Nivalda de Carvalho-Freitas é psicóloga, doutora em Administração pela Universidade Federal de Minas Gerais e professora adjunta do D epartamento e do Mestrado de Psicologia da Universidade Federal de São João delRei. Coordena o Núcleo de Pesquisas em Acessibilidade, Diversidade e Trabalho (NACE).

Contato com a autora:

Rua Felipe Marchetti, 80, Vila Marchetti, CEP: 36307-248. São João del-Rei - MG .

Email: nivalda@ufsj.edu.br 